\title{
Pituitary Adenylate Cyclase-Activating Polypeptide Is Required for the Development of Spinal Sensitization and Induction of Neuropathic Pain
}

\author{
Tamaki Mabuchi, ${ }^{1 \star}$ Norihito Shintani, ${ }^{2 \star}$ Shinji Matsumura, ${ }^{1}$ Emiko Okuda-Ashitaka, ${ }^{1}$ Hitoshi Hashimoto, ${ }^{2}$ \\ Tadatoshi Muratani, ${ }^{4}$ Toshiaki Minami, ${ }^{4}$ Akemichi Baba, ${ }^{2,3}$ and Seiji Ito ${ }^{1}$ \\ ${ }^{1}$ Department of Medical Chemistry, Kansai Medical University, Moriguchi 570-8506, Japan, ${ }^{2}$ Laboratory of Molecular Neuropharmacology, Graduate \\ School of Pharmaceutical Sciences, and ${ }^{3}$ Laboratory of Molecular Pharmacology, Graduate School of Medicine, Osaka University, Suita 565-0871, Japan, \\ and ${ }^{4}$ Department of Anesthesiology, Osaka Medical College, Takatsuki 569-8686, Japan
}

The prolonged sensitization of pain transmission after nerve injury by increasing excitability of spinal neurons and thereby promoting repair is an adaptive response of the body. The neuropeptide pituitary adenylate cyclase-activating polypeptide (PACAP) is widely distributed in the nervous system and implicated in neurotransmission, neural plasticity, and neurotrophic actions. Although PACAP is distributed in the spinal cord and dorsal root ganglia, a role of PACAP in pain responses remains essentially unknown. Here we show that mice lacking the PACAP gene (PACAP ${ }^{-1-}$ ) did not exhibit inflammatory pain induced by intraplantar injection of carrageenan or neuropathic pain induced by L5 spinal nerve transection, whereas they did retain normal nociceptive responses. Intrathecal administration of NMDA induced mechanical allodynia in wild-type mice, but not in PACAP ${ }^{-1-}$ mice. The NMDA-induced allodynia in PACAP ${ }^{-1-}$ mice was reproduced by simultaneous intrathecal injection of PACAP with NMDA. Concomitant with the increase in PACAP immunoreactivity after nerve injury, NADPH-dependent nitric oxide synthase (NOS) activity visualized by NADPH diaphorase histochemistry markedly increased in the superficial layer of the spinal cord of wild-type mice, which was not observed in PACAP ${ }^{-1-}$ mice. Simultaneous addition of PACAP and NMDA caused translocation of neuronal NOS from the cytosol to the membrane and stimulated NO production in vitro. These results demonstrate that PACAP might promote the functional coupling of neuronal NOS to NMDA receptors for both inflammatory and neuropathic pain to occur.

Key words: PACAP; knock-out mice; pain; NMDA; nitric oxide synthase; translocation

\section{Introduction}

Pituitary adenylate cyclase-activating polypeptide (PACAP) is a neuropeptide originally isolated from ovine hypothalamus based on its ability to stimulate adenylate cyclase in rat anterior pituitary cell cultures. PACAP exists in two biologically active forms, PACAP-38 and the C-terminally truncated PACAP-27, both of which share $68 \%$ homology with vasoactive intestinal peptide (VIP) at their N-terminal domain (Vaudry et al., 2000). PACAP is widely distributed in both the CNS and PNS and is implicated in neurobiological functions such as neurotransmission, neural plasticity, and neurotrophic actions (Vaudry et al., 2000; Hashimoto et al., 2002). In the spinal cord, PACAP-immunoreactive

Received March 17, 2004; revised July 12, 2004; accepted July 13, 2004.

This work was supported in part by grants from the programs Grants-in-Aid for Twenty-First Century Center of Excellence, Scientific Research on Priority Areas, Creative Scientific Research, and Exploratory Research from the Ministry of Education, Culture, Sports, Science, and Technology of Japan; Grants-in-Aid for Scientific Research (A), (B), and (C) from Japan Society for the Promotion of Science; and by grants from the Science Research Promotion Fund of the Japan Private School Promotion Foundation and Taisho Pharmaceutical Company.

*T.M. and N.S. contributed equally to this work.

Correspondence should be addressed to Dr. Seiji Ito, Department of Medical Chemistry, Kansai Medical University, 10-15 Fumizono, Moriguchi, 0saka 570-8506, Japan. E-mail:ito@takii.kmu.ac.jp.

DOI:10.1523/JNEUROSCI.0983-04.2004

Copyright $\odot 2004$ Society for Neuroscience $\quad$ 0270-6474/04/247283-09\$15.00/0 nerve fibers was detected in the superficial layer of the dorsal horn, and PACAP-immunoreactive cell bodies were present in dorsal root ganglia (DRG) (Moller et al., 1993; Zhang et al., 1995). This distribution has led to the suggestion that PACAP might serve as sensory transmitters and that it could play an important role in the modulation of pain transmission. In fact, several studies have been performed to evaluate the role of PACAP in animal pain models, but whether PACAP is nociceptive or anti-nociceptive is controversial (Dickinson and Fleetwood-Walker, 1999).

Glutamate neurotransmission is an essential component of many forms of experience-dependent plasticity, which is an important feature of nervous systems, especially for neural development, memory and learning, and pain. The emerging image of NMDA receptor complex shows that the similarity between mechanisms of plasticity in various systems is greater than their apparent differences (Kind and Neumann, 2001; Ji et al., 2003). The recent isolation and characterization of NMDA receptor complexes have identified numerous candidate molecules, including neuronal nitric oxide synthase (nNOS), that could have crucial roles in glutamate receptor signaling and plasticity (Husi et al., 2000; Husi and Grant, 2001). nNOS is normally a constitutive enzyme whose activity is thought to depend on increases in 
intracellular calcium after NMDA receptor activation. Nitric oxide (NO) is well known to be involved in nociceptive processing in the spinal cord (Meller and Gebhart, 1993). We recently found that NO production by nNOS is involved in maintenance of neuropathic pain and that inhibition of NO production alleviates neuropathic pain (Mabuchi et al., 2003). After inflammation or nerve transection, PACAP expression was upregulated in various parts of the nervous system, including the DRG (Zhang et al., 1995, 1996, 1998). Because potent and selective low-molecularweight PACAP antagonists have not yet been developed, the role of PACAP in pain responses under physiological and pathophysiological conditions has not been fully addressed by exogenously administered PACAP. Recently, Hashimoto et al. (2001) have generated mice deficient in PACAP $\left(\mathrm{PACAP}^{-1-}\right)$ and revealed that PACAP plays a new role in the regulation of psychomotor behaviors. In the present study, we demonstrate that inflammatory pain and neuropathic pain disappear in $\mathrm{PACAP}^{-1-}$ mice and that upregulation of PACAP in the superficial layer of spinal cord enhances the functional coupling of nNOS and NMDA receptors and then NO production, which is essential for chronic pain.

\section{Materials and Methods}

Behavioral studies. All animals conformed to the regulations of the Animal Care Committee of Kansai Medical University and received humane care in accordance with the guidelines of the ethics committee of the International Association for the Study of Pain (Zimmermann, 1983). $\mathrm{PACAP}^{-1-}$ mice were produced by a gene-targeting technique (Hashimoto et al., 2001). Thermal responses were assessed as latency to withdrawal from a radiant heat stimulus as described earlier (Mabuchi et al., 2003). Mechanical sensitivity was determined with calibrated von Frey filaments, using the up-down paradigm of Chaplan et al. (1994). The inflammatory pain model was made by intraplantar injection of $\lambda$-carrageenan $(0.4$ $\mathrm{mg} / 40 \mu \mathrm{l}$ ) into the left hindpaw (Doi et al., 2002). The neuropathic pain model was made as described previously (Mabuchi et al., 2003). Briefly, mice were anesthetized by intraperitoneal administration of sodium pentobarbital $(50 \mathrm{mg} / \mathrm{kg}$ ), and the left L5 spinal nerve was isolated, tightly ligated with $4-0$ silk thread at two locations (5 $\mathrm{mm}$ apart), and transected between ligatures. Control mice were subjected to sham operation with the same surgical procedure without nerve ligation and transection. Before and after intraplantar injection of carrageenan and L5 spinal nerve transection, thermal and mechanical thresholds were determined for both ipsilateral and contralateral paws.

Intrathecal injection and studies on allodynia were performed as described previously (Minami et al., 2001). Briefly, a 27 gauge stainless steel needle attached to a microsyringe was inserted between the L5 and L6 vertebrae, and drugs in saline $(5 \mu \mathrm{l})$ were injected slowly into the subarachnoid space of conscious mice. After the intrathecal injection, each mouse was placed in an individual $13 \times 8.5 \times 13 \mathrm{~cm}$ Plexiglas enclosure with wood chips on the floor, and then they were observed. Allodynia was assessed once every $5 \mathrm{~min}$ for $50 \mathrm{~min}$ after injection by light stroking of the flank of the mice with a paintbrush. The allodynic response was ranked as follows: 0, no response; 1, mild squeaking with attempts to move away from the stroking probe; 2, vigorous squeaking evoked by the stroking probe, biting at the probe, or used as a control. strong efforts to escape. The maximum possible score for allodynia of six mice was $2 \times 6=12$ in any $5 \mathrm{~min}$ and $2 \times 6 \times 10=120$ for a $50 \mathrm{~min}$ period.

Reverse transcription-PCR analysis. Wild-type and $\mathrm{PACAP}^{-1-}$ mice were decapitated before treatment, $6 \mathrm{hr}$ after intraplantar injection of carrageenan or $10 \mathrm{~d}$ after L5 spinal nerve transection. Right paw did not receive treatment. Total RNA extraction and reverse transcription-PCR were performed as described previously (Kitanaka et al., 1996) with the primers as follows: $5^{\prime}$-AGAAGACGAGGCTTACGACCAG-3' and $5^{\prime}$ TTTCTTGACAGCCATTTGTTTTCGG-3' for PACAP; 5'-CAGGAACCGGGAACAGACT-3' and 5'-TATCAGGAATGCCAGGAACT-3' for VIP; 5'-AGCTGCACTGTACCCGTAAC-3' and 5'-GCTGTGCTGTCATTCATATCC-3' for PAC ${ }_{1}$; 5'-TGCAGCAAGATGTGGGACAA-3' and $5^{\prime}$ TGTTGCTGCTCATCCATACT- $3^{\prime}$ for $\mathrm{VPAC}_{1} ; 5^{\prime}$-ATGGACAGCAACTCGCCTCTCTTTAG-3' and 5'-GGAAGGAACCAACACATAACTCAAACAG-3' for $\mathrm{VPAC}_{2}$; and $5^{\prime}$-GATGGTGGGTATGGGTCAGAAGGA-3' and $5^{\prime}$-GCTCATTGCCGATAGTGATGACCT- $3^{\prime}$ for $\beta$-actin. PCR reaction consisted of denaturation at $94^{\circ} \mathrm{C}$ for $1 \mathrm{~min}$, annealing at $60^{\circ} \mathrm{C}$ for $1 \mathrm{~min}$, and elongation at $72^{\circ} \mathrm{C}$ for $2 \mathrm{~min}$. The PCR products were analyzed with a fluorescent image analyzer (FluorImager 595; Molecular Dynamics, Tokyo, Japan). The numbers of cycles were optimized for each primer pair to obtain linearity between the amount of cDNA and PCR product. Product lengths (in base pair) and cycles are as follows: 343 and 30 for PACAP; 131 and 36 for VIP; 373 and 24 for $\mathrm{PAC}_{1} ; 221$ and 34 for $\mathrm{VPAC}_{1} ; 316$ and 32 for $\mathrm{VPAC}_{2}$; and 630 and 24 for $\beta$-actin.

For quantification of each gene, a real-time PCR assay was performed on a DNA Engine Opticon 2 (MJ Research) using the DyNAmo SYBR Green qPCR kit (FINNZYMES) and the same primers as described above except for $\mathrm{PAC}_{1} ; 5^{\prime}$-CAAGAAGGAGCAAGCCATGT-3' and 5'CTTGGTCCGGGTTGAAGATC-3' and glyceraldehyde-3-phosphate dehydrogenase (G3PDH); 5'-CTCATGACCACAGTCCATGC-3' and 5'-CACATTGGGGGTAGGAACAC-3'. The PCR parameters were one cycle of $95^{\circ} \mathrm{C}$ for $10 \mathrm{~min}$, followed by 40 cycles of denaturation at $95^{\circ} \mathrm{C}$ for
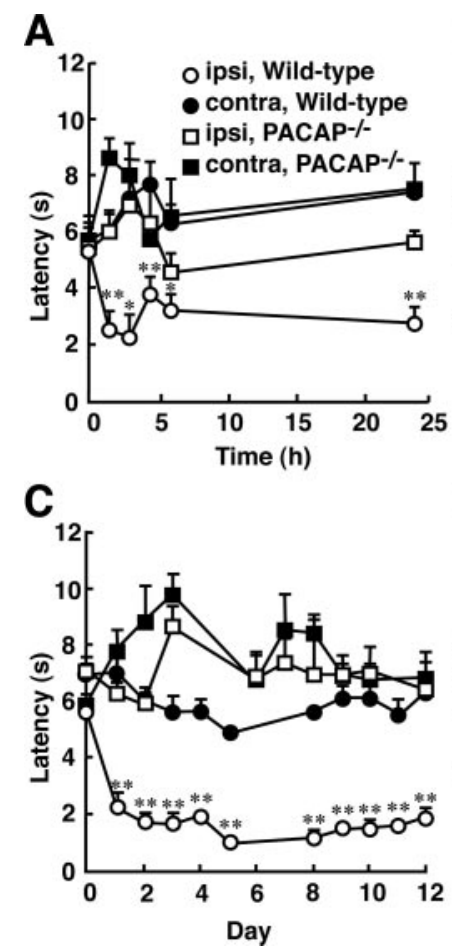

E
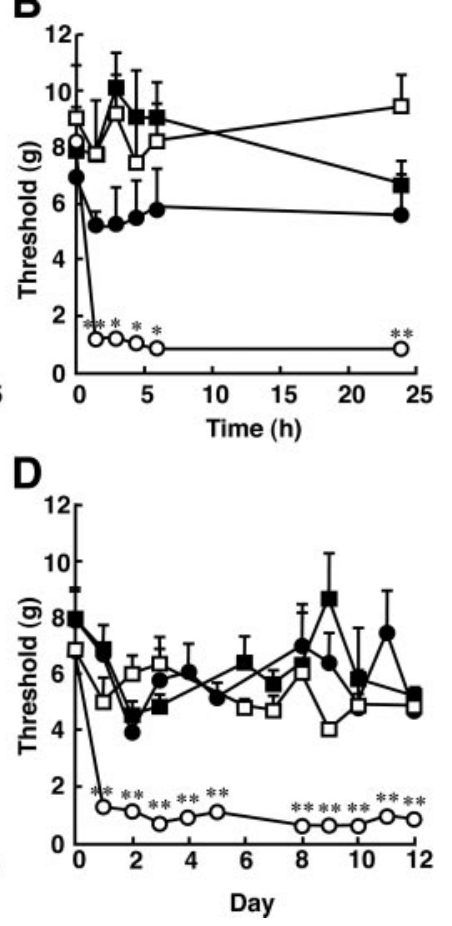
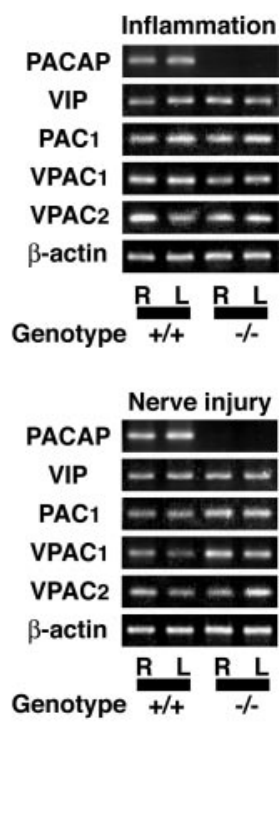

Figure 1. Loss of inflammatory pain and neuropathic pain in PACAP ${ }^{-1-}$ mice. $A-D$, Thermal hyperalgesia $(A, C)$ and mechanical allodynia $(B, D)$ after intraplantar injection of carrageenan $(A, B)$ or $\mathrm{L} 5$ spinal nerve transection $(C, D) .{ }^{*} p<0.05,{ }^{* *} p<0.01$ compared with the contralateral side. E, Expression of PACAP, VIP, and their receptors in the spinal cord. Total RNA was extracted from contralateral $(R)$ and ipsilateral $(\mathrm{L})$ sides of the $L 4-\mathrm{L} 5$ spinal cord from three mice $6 \mathrm{hr}$ after intraplantar carrageenan injection (Inflammation) and $10 \mathrm{~d}$ after L5 spinal nerve injury and subjected to reverse transcription-PCR analysis. $\beta$-Actin was 
$10 \mathrm{sec}$, annealing at $58^{\circ} \mathrm{C}$ for $20 \mathrm{sec}$, elongation at $72^{\circ} \mathrm{C}$ for $20 \mathrm{sec}$, and measuring the fluorescence of SYBR Green at $78^{\circ} \mathrm{C}$ for $2 \mathrm{sec}$. At the end of each program a melt-curve analysis was performed on a subset of the genes to ascertain specificity of amplification and purity of the PCR products. A threshold cycle value for each mRNA was determined using the Opticon Monitor software (version 2.02). All data were normalized to the G3PDH mRNA level and expressed as mRNA relative percentage change.

Histochemistry. Animals were anesthetized by intraperitoneal administration of sodium pentobarbital $(50 \mathrm{mg} / \mathrm{kg})$ and intracardially perfused with $50 \mathrm{ml}$ of physiological saline followed by a fixative containing $4 \%$ paraformaldehyde in $0.12 \mathrm{~m}$ sodium phosphate, $\mathrm{pH}$ 7.4. After dissection, the spinal cord and DRG were postfixed overnight in the same fixative at $4^{\circ} \mathrm{C}$ and then kept in $0.1 \mathrm{~m}$ sodium phosphate buffer, $\mathrm{pH} 7.4$, containing $30 \%(\mathrm{w} / \mathrm{v})$ sucrose for $1 \mathrm{~d}$. Transverse L5 spinal cord sections $(40 \mu \mathrm{m})$ were cut on a cryostat and processed for immunohistochemistry with anti-PACAP (1:6000; Yanaihara Institute, Shizuoka, Japan) or antinNOS (1:2000; Santa Cruz Biotechnology, Santa Cruz, CA) rabbit polyclonal antibody, and Cy3-conjugated goat anti-rabbit IgG (1:300; Jackson ImmunoResearch, West Grove, PA) as primary and secondary antibodies, respectively. Frozen DRG were cut on a cryostat in $8 \mu \mathrm{m}$ sections that were collected on silane-coated glass slides. For triple immunofluorescence, DRG sections were serially incubated with antiPACAP antibody (1:6000) and Cy3-conjugated goat anti-rabbit IgG (1:
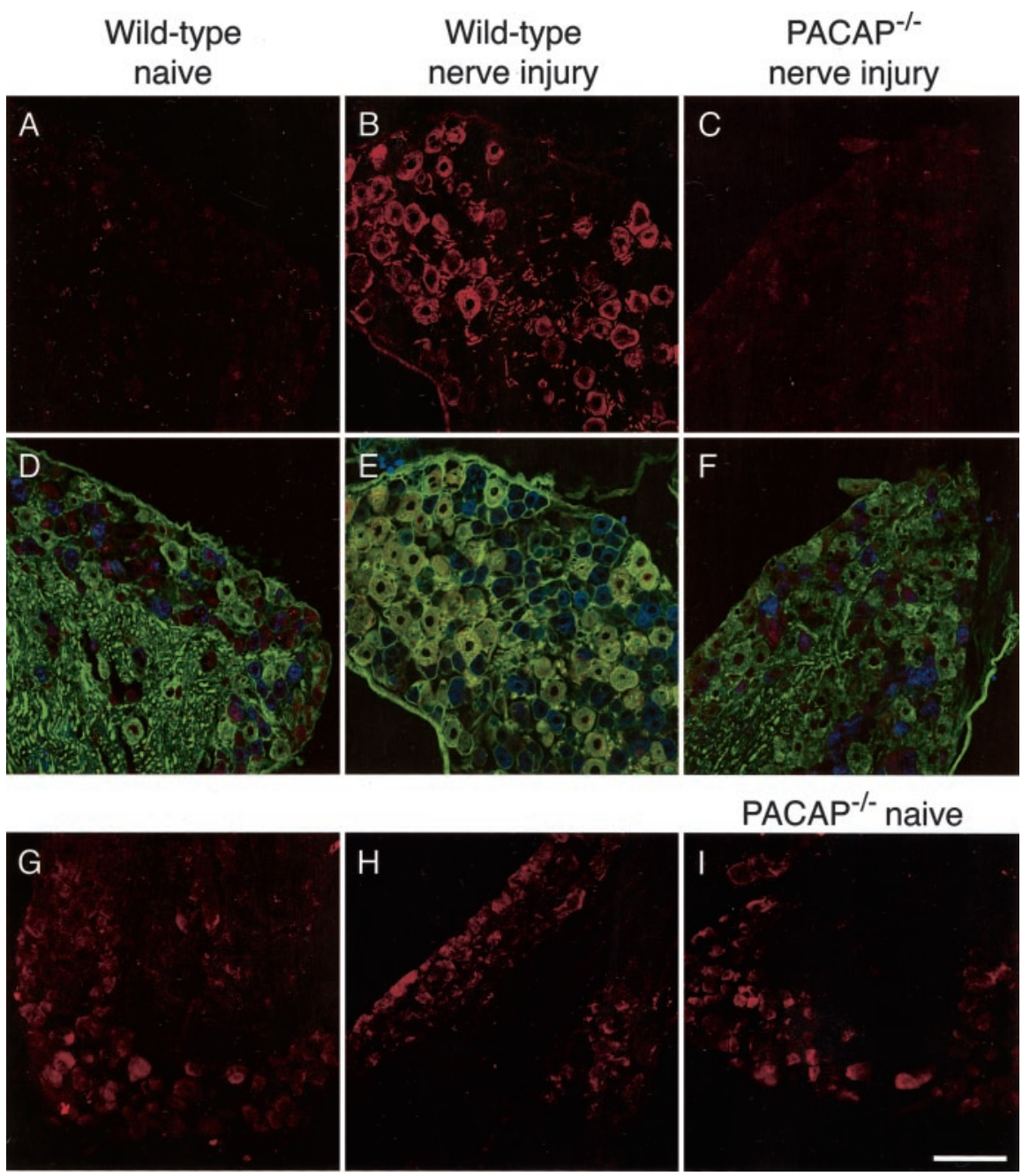

Figure 2. Upregulation of PACAP immunoreactivity in DRG after nerve transection. $A-F$, Expression of PACAP immunoreactivity in DRG from naive wild-type mice $(A, D), L 5$ nerve-transected wild-type mice $(B, E)$, and $L 5$ nerve-transected PACAP ${ }^{-1-}$ mice $(C, F) . G-I$, Expression of VIP immunoreactivity in L5 DRG from naive wild-type mice $(G), L 5$ nerve-transected wild-type mice $(H)$, and naive PACAP $^{-I-}$ mice (I). L5 DRG sections ( $8 \mu \mathrm{m}$ thick) ipsilateral to nerve transection were immunostained with antiPACAP, anti-NF200, and anti-VIP antibodies or labeled with IB4 as described in Materials and Methods. Triple labeling $(D-F)$ was created by merging the images obtained for PACAP (red), NF200 (green), and IB4 (blue) together. Scale bar, $100 \mu \mathrm{m}$.
300) for PACAP, anti-neurofilament 200 (NF200) monoclonal antibody (1:400; Sigma, St. Louis, MO), and Cy5-conjugated donkey anti-mouse IgG (1:300; Jackson ImmunoResearch) for NF200, and then FITCconjugated isolectin B4 (IB4) (1:400; Sigma) for IB4 lectin-binding histochemistry. Anti-VIP rabbit polyclonal antibody (1:400; Diain, Stillwater, MN) was used as primary antibody for VIP immunohistochemistry. Digital images were captured on a Zeiss laserscanning confocal microscope (LSM510) equipped with argon (excitation at $488 \mathrm{~nm}$ for FITC), HeNe1 (at $543 \mathrm{~nm}$ for Cy3), and $\mathrm{HeNe} 2$ (at 633 $\mathrm{nm}$ for Cy5) lasers and the appropriate filters. Translocation of nNOS in nerve growth factor (NGF)-differentiated pheochromocytoma cell line (PC12) cells was examined as reported (Arundine et al., 2003). After a 30 min incubation with PACAP and/or NMDA, the cells were fixed and processed for immunohistochemistry with anti-nNOS antibody (1:6000; Santa Cruz Biotechnology). The intensity of nNOS immunoreactivity was quantified using NIH Image. Distribution of NOS activity was determined using NADPH diaphorase histochemistry as described previously (Mabuchi et al., 2003). The incubation was performed for $2.5 \mathrm{hr}$ at $37^{\circ} \mathrm{C}$.

Fluorometric measurement. Lumbosacral segments prepared from C57BL/6 mice were cut using a vibrating blade microtome (VT-1000S; Leica, Nussloch, Germany), and slices were incubated for $2 \mathrm{hr}$ in an artificial CSF bubbled with $95 \% \mathrm{O}_{2}$ and $5 \% \mathrm{CO}_{2}$ at $37^{\circ} \mathrm{C}$. The slices $(350$ $\mu \mathrm{m}$ thick) thus obtained from lumbar segments L4-L6 were used for fluorometric measurements. $\left[\mathrm{Ca}^{2+}\right]$ i in fura-2loaded slices was measured as a fluorescence ratio obtained by excitation at 340 and $380 \mathrm{~nm}$ as described previously (Minami et al., 2003). NO generation in spinal slices and PC12 cells was measured with diaminofluorescein (DAF)-FM as fluorescence intensity obtained by excitation at $480 \mathrm{~nm}$ essentially as described earlier (Mabuchi et al., 2003).

Western blot analysis. Translocation of nNOS protein in NGF-differentiated PC12 cells was examined as reported (Arundine et al., 2003) with anti-nNOS rabbit polyclonal antibody (1: 6000; Santa Cruz Biotechnology) using ECL Western blotting detection reagents (Amersham Biosciences, Piscataway, NJ).

Statistics. Statistical analyses of the results were made by Student's $t$ test, Student's paired $t$ test, or the Mann-Whitney $U$ test.

\section{Results}

Disappearance of inflammatory pain and neuropathic pain in PACAP $^{-1-}$ mice

$\mathrm{PACAP}^{-1-}$ mice were born in the expected Mendelian ratios (Hashimoto et al., 2001), and there was no measurable difference in thermal and mechanical nociception between naive wild-type and PACAP $^{-1-}$ mice. The latency to thermal stimuli was $5.55 \pm 0.48$ and $5.61 \pm 0.58 \mathrm{sec}$ in wild-type and $\mathrm{PACAP}^{-1-}$ mice, respectively (Fig. 1A). The two groups of mice, however, showed a marked difference in nociceptive thresholds after the intraplantar injection of carrageenan. Whereas the thermal latency was reduced in the hindpaw on the ipsilateral $(2.57 \pm 0.62 \mathrm{sec})$, but not contralateral $(6.07 \pm 0.66 \mathrm{sec})$, side of wild-type mice at $90 \mathrm{~min}$ after the injection and remained low $(2.77 \pm 0.56$ $\mathrm{sec})$ at $24 \mathrm{hr}$ in them, it did not decrease in the PACAP ${ }^{-1-}$ mice (Fig. $1 A$ ). Conspicuous difference was observed in the me- 
chanical threshold between wild-type and $\mathrm{PACAP}^{-1-}$ mice after carrageenan injection (Fig. $1 B$ ), suggesting that PACAP is involved in inflammatory pain.

To clarify the involvement of PACAP in neuropathic pain, we used an L5 spinal nerve transection model (Mabuchi et al., 2003). Wild-type mice showed persistent thermal hyperalgesia and mechanical allodynia of the injured paw 1-12 d after the nerve transection. The withdrawal latency to thermal stimuli was reduced on the ipsilateral side from $5.53 \pm 0.74 \mathrm{sec}$ before operation to $2.15 \pm 0.63 \mathrm{sec}$ on day 1 after operation, and this hyperalgesic state continued throughout the experimental period (Fig. 1C). Similarly, the threshold to mechanical stimuli was dramatically reduced on the injured side in wild-type mice (Fig. 1D). Similar to the noninjured paws of wild-type mice, the injured paws of PACAP $^{-1-}$ mice showed neither thermal hyperalgesia nor mechanical allodynia (Fig. 1C,D). As expected, the PCR product of PACAP was completely absent in $\mathrm{PACAP}^{-/-}$mice (Fig. 1E). To quantify the expression mRNA levels in the spinal cord, we performed real-time PCR. PACAP mRNA of wild-type spinal cord was significantly increased in the ipsilateral side as compared with the contralat-
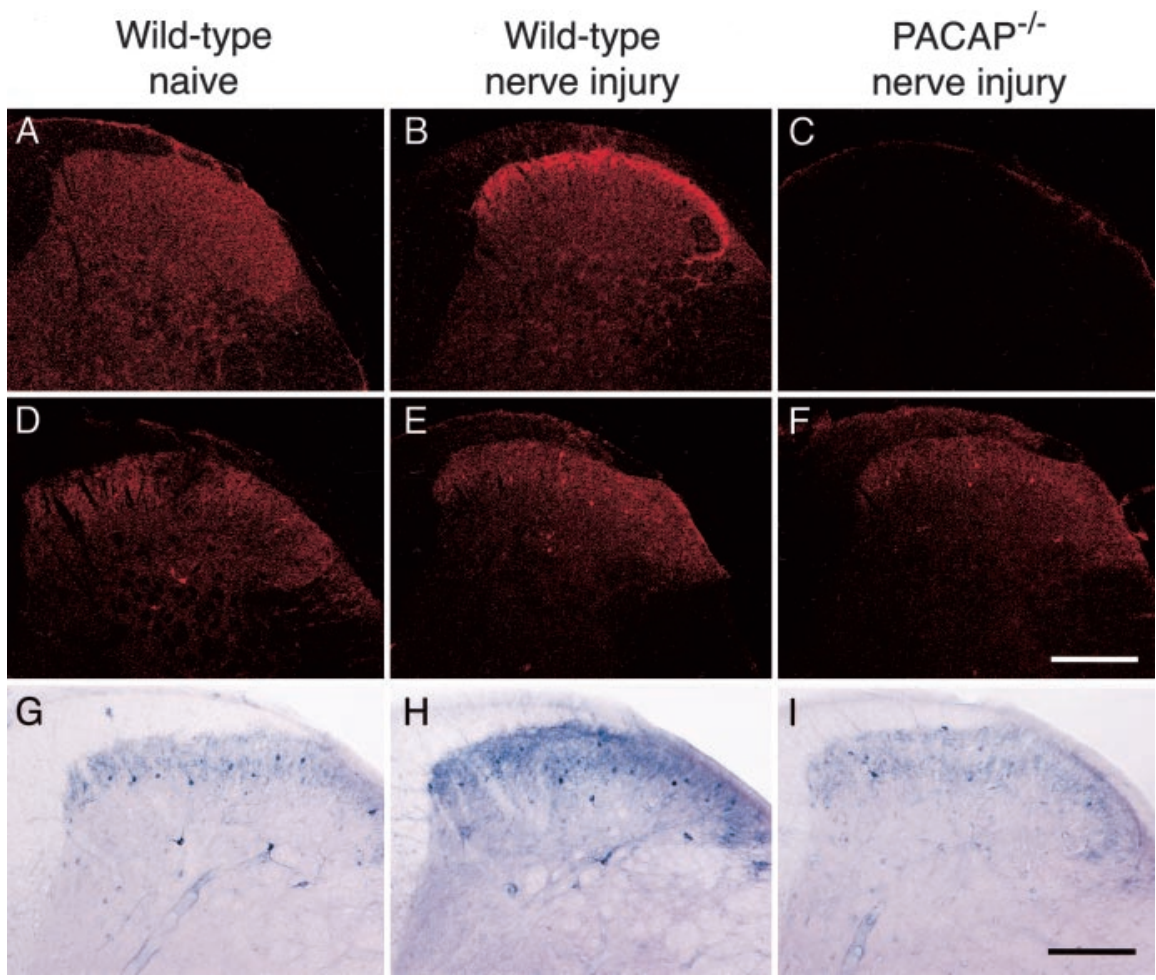

I

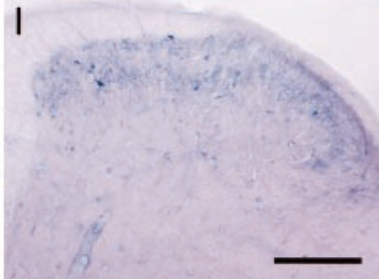

Figure 3. Increase in $n N O S$ activity by upregulation of PACAP after $L 5$ nerve transection. Spinal slices prepared from naive wild-type mice $(A, D, G), \mathrm{L} 5$ nerve-transected wild-type mice $(B, E, H)$, and $\mathrm{L} 5$ nerve-transected PACAP ${ }^{-1-}$ mice $(C, F, I)$ were immunostained with anti-PACAP $(A-C)$ or anti-nNOS $(D-F)$ antibodies or stained for $2.5 \mathrm{hr}$ with NADPH diaphorase $(G-l)$. Scale bars, $200 \mu \mathrm{m}$. eral side after carrageenan injection $(248 \pm 48 \% ; n=6-7)$ and after nerve transection $(230 \pm 36 \%$; $n=4-6)$. However, the difference in the expression of VIP or the receptors $\mathrm{PAC}_{1}, \mathrm{VPAC}_{1}$, and $\mathrm{VPAC}_{2}$ in the spinal cord was not statistically significant between naive and treated mice, between ipsilateral and contralateral sides after the carrageenan injection or after the nerve transection, nor between wild-type and PACAP $^{-1-}$ mice by real-time PCR (data not shown). These results suggest that PACAP is indispensable for the development of neuropathic pain as well as inflammatory pain.

\section{Expression of PACAP immunoreactivity in DRG and spinal cord}

To clarify the role of PACAP in pain transmission, the expression and distribution of PACAP were examined in the DRG and spinal cord of wild-type and PACAP ${ }^{-1-}$ mice. Whereas PACAP immunoreactivity was weakly observed in the DRG of naive wild-type mice (Fig. $2 A$ ), the intensity of immunostaining was markedly increased in the L5 DRG (Fig. 2B) as well as in the L4 DRG (data not shown) $7 \mathrm{~d}$ after L5 spinal nerve transection. IB4 lectin (blue) and NF200 antibody (green) were used to label unmyelinated C-fiber and myelinated A-fiber DRG cell population, respectively (supplemental Fig. 1, available at www.jneurosci.org/cgi/content/ full/24/34/7283/DC1). Whereas PACAP-positive cells were sparsely colabeled with IB4-positive small-sized neurons in naive mice (Fig. $2 D$ ), numerous PACAP-positive neurons were colabeled with NF200, but not with IB4 after L5 spinal nerve transection (Fig. 2E), demonstrating that myelinated neurons began to express PACAP in the DRG after nerve injury. These changes are consistent with a previous report showing that middle-sized and large DRG neurons became PACAP-positive $10 \mathrm{~d}$ after axotomy (Zhang et al., 1995). On the other hand, the expression of VIP was weak in the DRG of naive mice and did not increase in the DRG $7 \mathrm{~d}$ after nerve transection (Fig. $2 G, H)$. PACAP immunoreactivity was absent in PACAP $^{-1-}$ mice (Fig. 2C), and the expression of VIP did not change markedly between wild-type and PACAP ${ }^{-1-}$ mice (Fig. 2G,I).

In the spinal cord, although it was faintly detected over the spinal cord of naive wild-type mice, PACAP immunoreactivity was dense in the superficial layer, especially laminas I-II of the dorsal horn, and was scattered in the deeper layer of the dorsal horn on day 7 after nerve transection (Fig. $3 A, B$ ). On the other hand, PACAP immunoreactivity was completely absent in the spinal cord of $\mathrm{PACAP}^{-1-}$ mice regardless of the surgery (Fig. $3 C$ ). In contrast to PACAP, VIP immunoreactivity was very weak in the spinal cord and did not significantly change after nerve transection (data not shown).

\section{Increase in NO production in the spinal cord associated with neuropathic pain}

There is abundant evidence for the role of NO of neurogenic origin in spinal processing of nociceptive input (Meller and Gebhart, 1993; Mabuchi et al., 2003). To clarify the relationship between NO production and the disappearance of neuropathic pain in PACAP ${ }^{-/-}$mice, we examined the distribution of nNOS protein and NOS activity in the spinal cord. nNOS immunoreactivity was detected in the laminas I-III, and there was no significant difference in intensity between naive and treated wild-type mice (Fig. $3 D, E$ ) or between wild-type and PACAP $^{-1-}$ mice (Fig. $3 E, F$ ) after nerve injury. In contrast, when NOS activity was assessed in terms of NADPH diaphorase activity, a reliable marker of nNOS activity in the spinal cord (Laing et al., 1994), the number of NADPH diaphorase-active neurons and the intensity of staining in neuropiles evidently increased in the dorsal horn, especially in the superficial layer, of neuropathic mice (Fig. $3 \mathrm{H}$ ) 


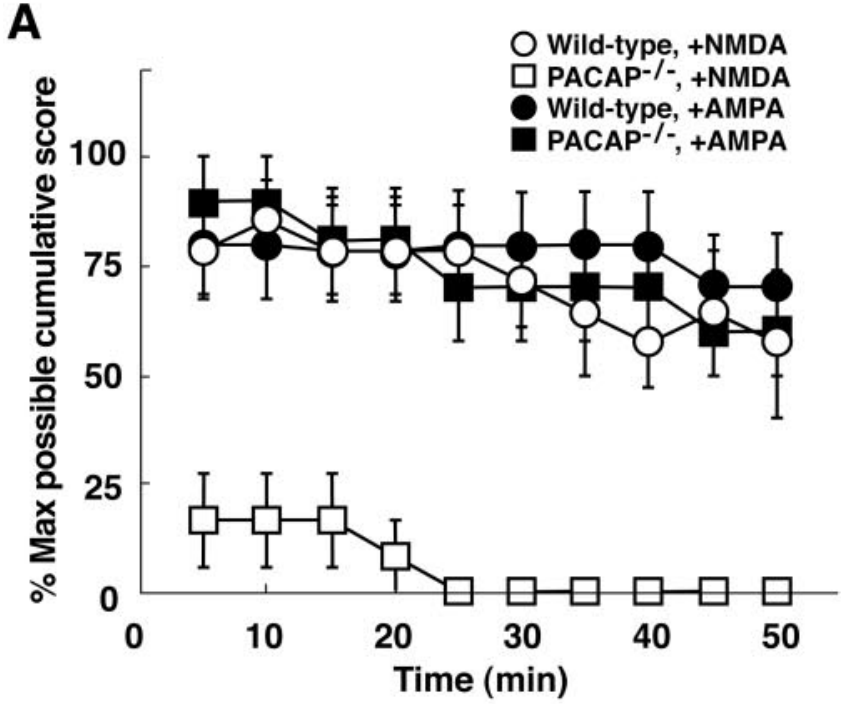

B

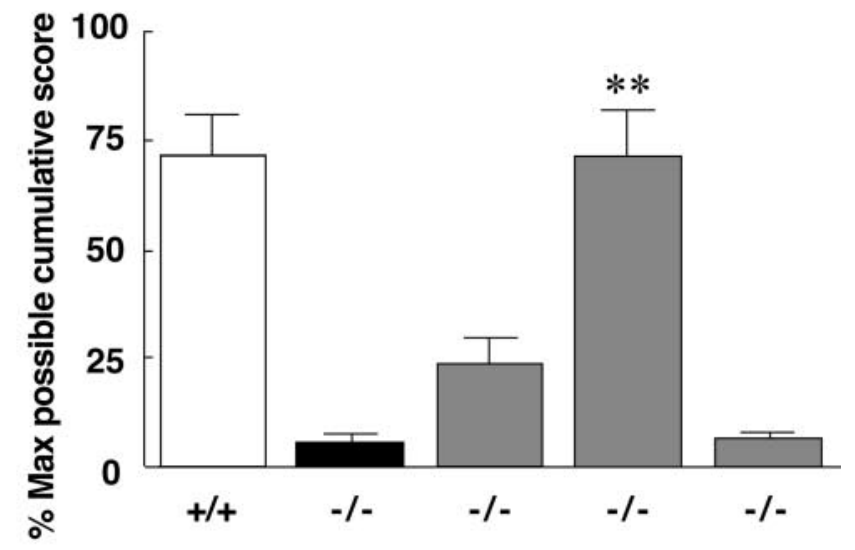

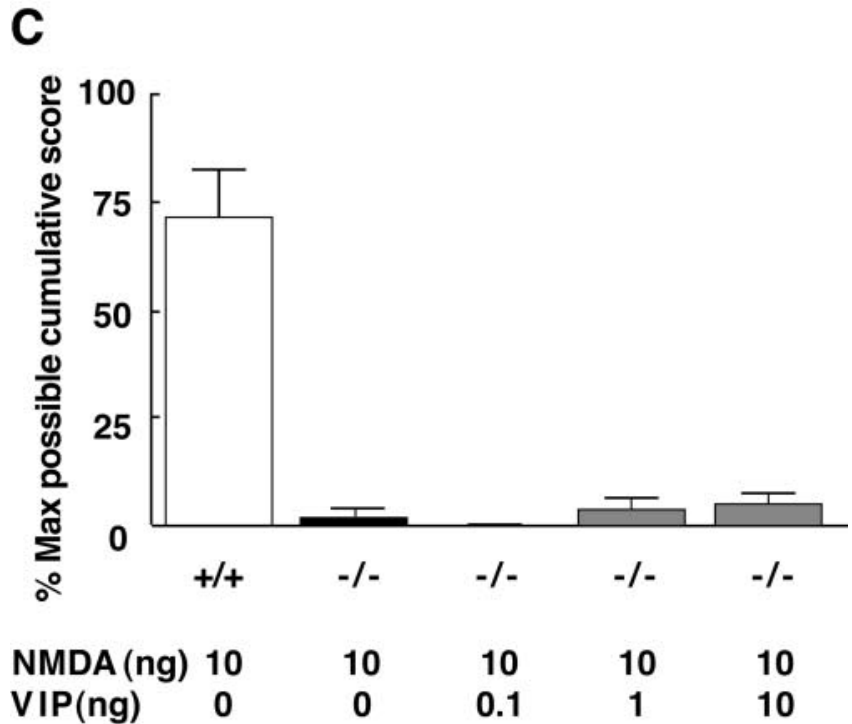

Figure 4. Synergism of NMDA and PACAP for induction of allodynia in PACAP ${ }^{-1-}$ mice. $A$, Mechanical allodynia induced by intrathecal injection of NMDA (10 ng) or AMPA (10 ng). Allodynia was assessed once every $5 \mathrm{~min}$ as described in Materials and Methods. Each column as compared with those in naive mice (Fig. 3G) and PACAP ${ }^{-1-}$ mice after nerve injury (Fig. 3I).

Recovery of NMDA-induced allodynia by PACAP in PACAP $^{-/-}$mice

nNOS is a constitutive enzyme whose activity is thought to depend on an increase in the level of intracellular calcium after NMDA receptor activation. We previously showed that intrathecal administration of NMDA or AMPA induced allodynia (Minami et al., 2001). Whereas AMPA (10 ng/mouse) induced allodynia in both wild-type and PACAP ${ }^{-1-}$ mice over a $50 \mathrm{~min}$ experimental period, NMDA ( $10 \mathrm{ng} /$ mouse) could induce allodynia in wild-type mice, but not in $\mathrm{PACAP}^{-1-}$ mice (Fig. 4A). When the scores of allodynia obtained for the overall 50 min were cumulated, the scores evoked by 10 ng NMDA were markedly reduced from $71.4 \pm 10.8 \%$ in wild-type mice to $5.8 \pm 2.4 \%$ in PACAP $^{-1-}$ mice (Fig. 4B). Simultaneous intrathecal injection of PACAP with 10 ng of NMDA evoked allodynia in PACAP $^{-1-}$ mice in a bell-shaped manner with a peak at $1 \mathrm{ng}$, the extent of which was comparable to that induced by NMDA alone in wildtype mice (Fig. 4B). On the other hand, intrathecal VIP did not restore NMDA-induced allodynia in PACAP ${ }^{-1-}$ mice (Fig. 4C). These results demonstrate that endogenous PACAP may be involved in mechanical allodynia associated with neuropathic pain.

\section{Stimulation of NO formation in the spinal cord by PACAP and NMDA}

Recently, a new fluorometric method for direct detection of NO formation has been developed with a fluorescent $\mathrm{NO}$ indicator DAF-FM (Kojima et al., 1999). To clarify the involvement of NO formation by PACAP in recovery of NMDA-induced allodynia in $\mathrm{PACAP}^{-1-}$ mice, we fluorometrically examined $\mathrm{NO}$ formation in the lumbar spinal cord of naive mice. NO formation was monitored in three regions; the laminas I-II (black), laminas III-IV (red), and lamina V (blue), of a DAF-FM-loaded dorsal horn (Fig. $5 A$ ). Figure $5 B-D$ illustrates a representative of time courses of NO formation in the dorsal horn of the spinal cord and fluorescent images before and after stimulation. Although NMDA $(100 \mu \mathrm{M})$ alone could produce a rapid and transient increase in $\left[\mathrm{Ca}^{2+}\right] \mathrm{i}$ in the dorsal horn, it did not increase NO formation in the superficial layer or in the deeper layer (Fig. $5 B$ ). On the other hand, PACAP (50 nM) alone weakly increased the fluorescence intensity in the dorsal horn, and simultaneous addition of $50 \mathrm{~nm}$ PACAP and $100 \mu \mathrm{M}$ NMDA prominently increased both in the superficial layer and in the deeper layer of the dorsal horn (Fig. $5 C, D)$. Figure $5 E$ summarizes the dose dependency of PACAP for NO formation using 63 lumbar spinal slices prepared from 23 mice. PACAP increased the fluorescence intensity in a dosedependent manner with a maximum effect at $10 \mathrm{~nm}$.

Translocation of nNOS from the cytosol to the membrane by PACAP and NMDA

PC12 cell line was initially developed to study cell differentiation and has been extensively used for studies on PACAP. nNOS is

$\leftarrow$

(mean $\pm \mathrm{SEM}$ ) represents the percentage of the maximum possible cumulative score of six mice evaluated every $5 \mathrm{~min}$. The maximum possible cumulative score for allodynia in any $5 \mathrm{~min}$ period was 12 and was taken as $100 \% . B, C$, Restoration of NMDA-induced allodynia by intrathecal PACAP in PACAP ${ }^{-1-}$ mice. The indicated doses of PACAP $(B)$ or VIP $(C)$ were simultaneously injected singly with $10 \mathrm{ng}$ of NMDA into PACAP ${ }^{-1-}$ mice. Data (mean $\pm \mathrm{SEM} ; n=6$ ) are cumulated over a 50 min period and expressed as a percentage of the maximum possible cumulative score over this period. ${ }^{* *} p<0.01$ compared with PACAP ${ }^{-1-}$ mice without PACAP injection. 
A
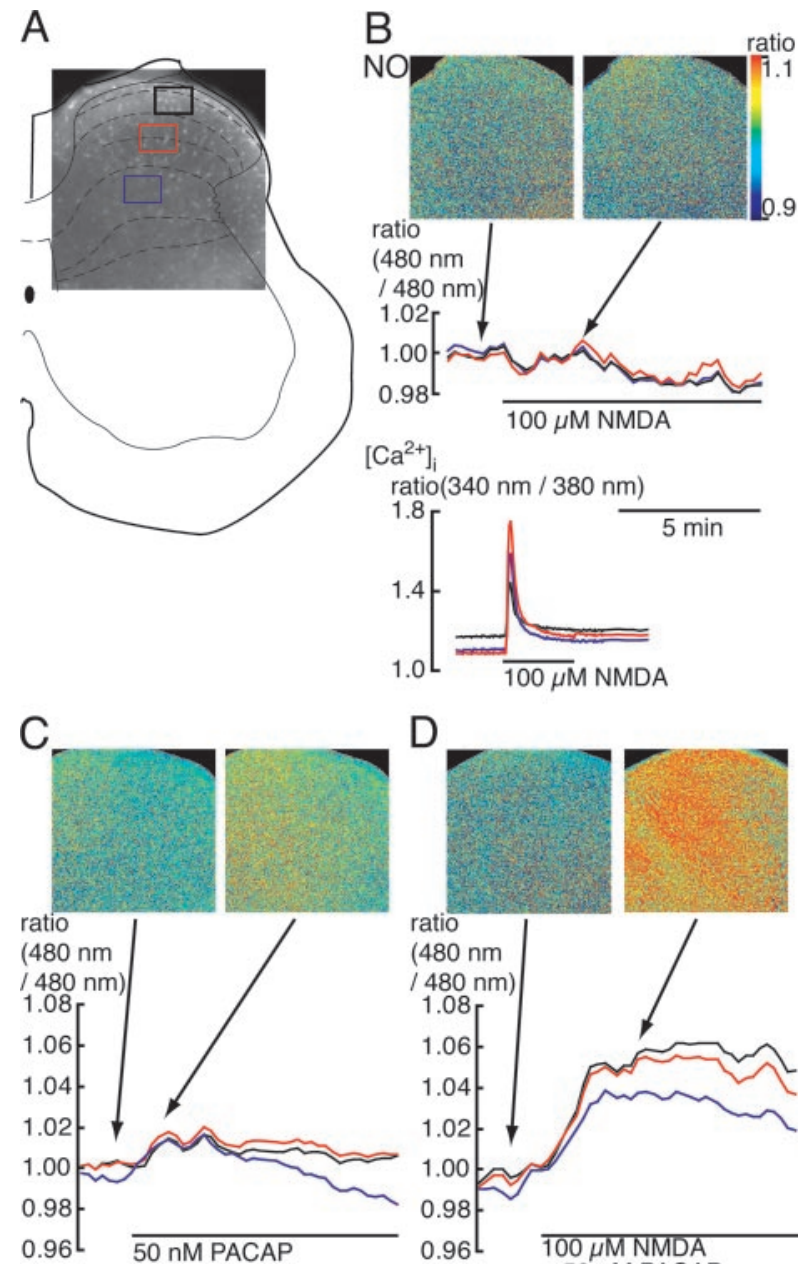

$\mathrm{E}$

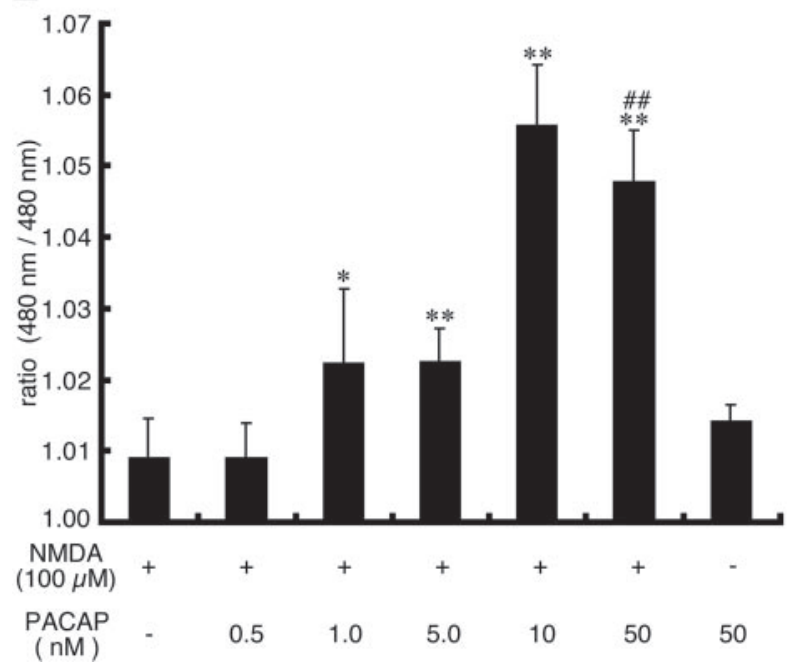

Figure 5. NO formation by PACAP in spinal cord slices prepared from wild-type mice. A, Schematic presentation of spinal transverse section at $L 5$ level. Broken lines indicate laminas in DAF-FM-loaded dorsal horn and boxes depict the regions to monitor fluorescence images excited at $480 \mathrm{~nm} . B-D, N 0$ formation in DAF-FM-loaded spinal slices was measured at 15 sec intervals in the presence of $100 \mu \mathrm{m}$ $\operatorname{NMDA}(B), 50 \mathrm{~nm} \operatorname{PACAP}(C)$, or $100 \mu \mathrm{m} \operatorname{NMDA}$ plus $50 \mathrm{~nm} \operatorname{PACAP}(D)$. Fluorescence images are shown in pseudocolor as ratio images at the indicated time on the basis of the initial intensity at the start of measurement. B, Bottom panel, $\left[\mathrm{Ca}^{2+}\right] \mathrm{i}$ change by $100 \mu \mathrm{m}$ NMDA in fura-2-loaded spinal slice. $E$, Dose dependency of PACAP for NO formation. NO formation induced by PACAP is expressed as a ratio of the peak intensity obtained by the indicated doses of PACAP to the initial intensity. Each column represents the mean \pm SEM $(n=6-13) .{ }^{*} p<0.05 ;{ }^{* *} p<0.01$ compared with $100 \mu$ MMDA alone; ${ }^{\# \#} p<0.01$ compared with $50 \mathrm{~nm}$ PACAP alone. induced in PC12 cells by NGF (Sheehy et al., 1997). To clarify how PACAP activated nNOS activity in the spinal cord, we examined the effect of PACAP on nNOS sublocalization in NGFdifferentiated PC12 cells. nNOS was observed throughout the cytosol, with a few punctate regions on the membrane (Fig. 6A). Whereas the distribution of nNOS was not altered by either $1 \mathrm{nM}$ PACAP or $100 \mu \mathrm{M}$ NMDA alone, nNOS was markedly translocated to the membrane by simultaneous stimulation with $100 \mu \mathrm{M}$ NMDA and 1 nM PACAP (Fig. 6A). Translocation of nNOS protein from the cytosol to the membrane was confirmed by Western blot analysis (Fig. 6B). Immunoblot analysis of NGFdifferentiated PC12 cell lysates detected an nNOSimmunoreactive band with $160 \mathrm{kDa}$, the same position as nNOS protein in the cerebellum. Although it was predominantly localized in the soluble fraction in unstimulated control cells, nNOS immunoreactivity was mainly detected in the particulate fraction 30 min after stimulation of $1 \mathrm{~nm}$ PACAP and $100 \mu \mathrm{M}$ NMDA. NO formation associated with nNOS translocation was further confirmed in the cells loaded with DAF-FM. In agreement with the translocation of nNOS to the membrane (Fig. 6A), NO formation was observed in the cells treated by $1 \mathrm{nM}$ PACAP and $100 \mu \mathrm{M}$ NMDA, particularly close to the membrane (Fig. 6C). Significant NO formation was not detected in the PC12 cells treated by 100 $\mu \mathrm{M}$ NMDA or $1 \mathrm{~nm}$ PACAP alone. These results suggest that the functional coupling of nNOS and NMDA receptor occurs after translocation of nNOS from the cytosol to the membrane induced by PACAP and glutamate in the spinal cord.

\section{Discussion}

Although the constitutive level of PACAP was relatively low in the DRG and the spinal cord, PACAP immunoreactivity was markedly increased in the DRG (Fig. $2 A, B$ ) and laminas I-II of the spinal cord (Fig. 3A,B) $7 \mathrm{~d}$ after L5 spinal nerve transection. Consistent with the present study, Zhang et al. (1995) previously reported that transection of sciatic nerve induced a rapid and strong upregulation in PACAP mRNA in the DRG and that PACAP immunoreactivity was accumulated proximal to a ligature indicating axonal transport of the peptide from the DRG. There was a shift of PACAP expression from small-sized neurons to medium-sized and large-sized neurons in the DRG on the injured side (Fig. 2D,E) (Zhang et al., 1995), and PACAPimmunoreactive nerve fibers were also found on the ipsilateral side in the deeper laminas of the spinal cord (Fig. 3B), which might be important for neuropathic pain. Significant increase in PACAP mRNA was also observed in the spinal cord of wild-type mice after inflammation and L5 spinal nerve transection by realtime PCR. We demonstrated here that inflammatory pain and neuropathic pain did not occur in $\mathrm{PACAP}^{-1-}$ mice after intraplantar injection of carrageenan and after L5 spinal nerve transection (Fig. $1 A-D$ ). However, nociceptive responses to thermal and mechanical stimuli did not change, but rather delayed in PACAP $^{-1-}$ mice (Fig. $1 A-D$ ), the significance of which remains to be clarified. PACAP exerts the action via three heptahelical G-protein-linked receptors: one PACAP-specific $\left(\mathrm{PAC}_{1}\right)$ receptor and two receptors that it shares with VIP $\left(\mathrm{VPAC}_{1}\right.$ and $\mathrm{VPAC}_{2}$ ). As observed in the present study (Fig. $1 E$ ), $\mathrm{PAC}_{1}$, $\mathrm{VPAC}_{1}$, and $\mathrm{VPAC}_{2}$ receptors were constitutively expressed in the superficial layer of the spinal cord in naive animals, and their mRNA levels or number of positive cells in the dorsal horn did not differ between the transected and the uninjured sides after nerve injury (Dickinson et al., 1999; Jongsma et al., 2000). Previous studies on a role by intrathecal injection of PACAP in pain responses were controversial: anti- or pro-nociceptive (Zhang et 
A

Control
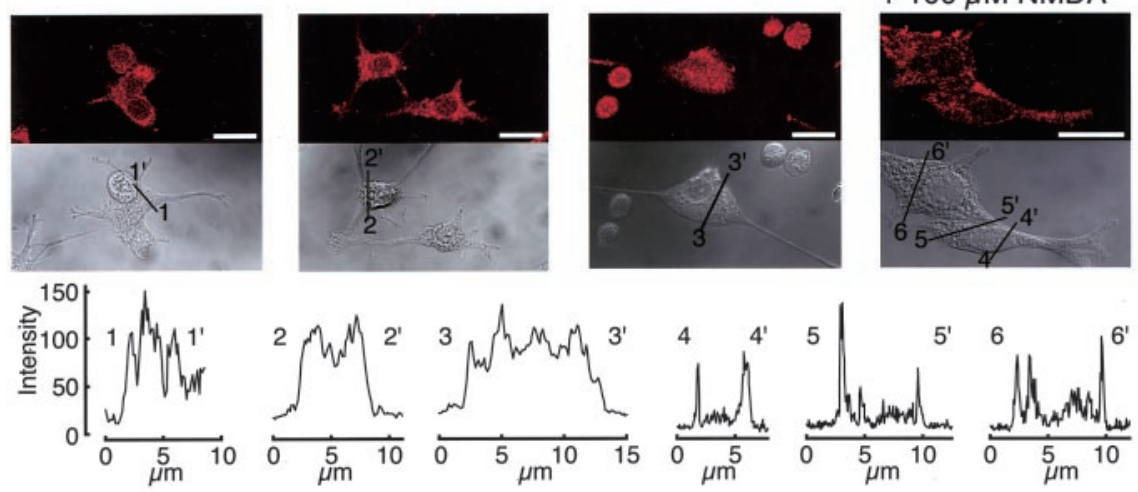

B

\section{Control}

\section{1 nM PACAP}

$+100 \mu \mathrm{M}$ NMDA

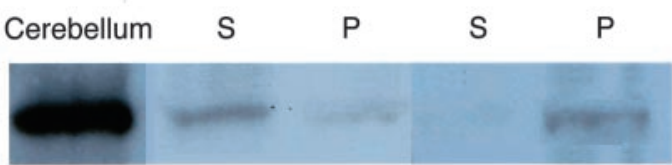

\section{C}

1 nM PACAP

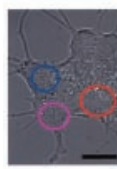

ratio $(480 \mathrm{~nm} / 480 \mathrm{~nm})$

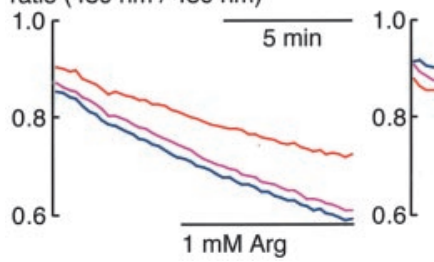
$100 \mu \mathrm{M}$ NMDA
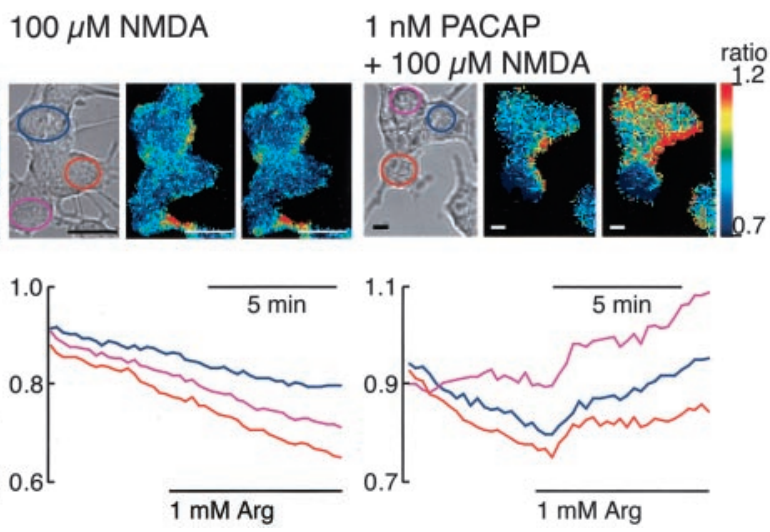

Figure 6. Functional coupling of NMDA receptor and nNOS by PACAP in PC12 cells. A, Translocation of nNOS by PACAP and NMDA. After a 30 min incubation without or with $1 \mathrm{~nm}$ PACAP and/or $100 \mu \mathrm{m}$ NMDA, NGF-differentiated PC12 cells were immunostained with anti-nNOS antiserum. The fluorescence intensity of nNOS was quantified along the line (1.68 $\mu \mathrm{m}$ width) using NIH Image and expressed with a scale of $0-255$. B. Western blot analysis of nNOS protein in $\mathrm{PC} 12$ cells. Lysates from cells without or with 30 min treatment of $1 \mathrm{~nm}$ PACAP and $100 \mu \mathrm{M}$ NMDA were subfractionated into soluble $(S)$ and particulate (P) fractions. nNOS immunoreactivity with a molecular weight of $160 \mathrm{kDa}$ was shifted from the soluble fraction to the membrane fraction by PACAP and NMDA. C, NO production induced by PACAP and NMDA in DAF-FM-loaded PC12 cells. After preincubation with $1 \mathrm{~nm} \mathrm{PACAP}$ and/or $100 \mu \mathrm{MNMDA}$, cells were loaded with DAF-FM for $30 \mathrm{~min}$. After washing of the cells with a recording buffer for $10-20$ min, $1 \mathrm{~mm} \mathrm{~L}$-arginine (Arg) was added to the recording buffer, and NO formation was fluorometrically measured. Scale bars, $10 \mu \mathrm{m}$.

may depend on doses used. Taken together, the present study clearly demonstrates that increase in PACAP levels in the dorsal horn, especially in laminas I and II after peripheral noxious stimuli may be crucial for inflammatory and neuropathic pain.

Glutamate is a fast excitatory neurotransmitter in the CNS. Despite extensive evidence to implicate spinal NMDA receptors and subsequent NO production in pain responses (Meller and Gebhart, 1993), little was known whether persistent activation of NMDA receptors is necessary for maintenance of chronic pain. We recently demonstrated that the increase in NOS activity after L5 nerve transection was observed in the superficial layer of the dorsal horn by NADPH diaphorase histochemistry and confirmed NO production by the fluorescent $\mathrm{NO}$ detection dye DAF-FM (Mabuchi et al., 2003). Because inducible NOS-deficient mice developed neuropathic pain, we concluded that inducible NOS is not involved in NO production in the L5 spinal nerve transection model. This notion was supported by the observation that the increase in NO production associated with nerve transection was reverted to the basal level concomitant with attenuation of neuropathic pain (Mabuchi et al., 2003). Here we showed that NOS activity in the dorsal horn visualized by NADPH diaphorase staining was well correlated with the development of neuropathic pain in wild-type and PACAP ${ }^{-/-}$ mice. Although PACAP staining completely disappeared in $\mathrm{PACAP}^{-/-}$mice, nNOS immunoreactivity did not differ between wild-type and PACAP $^{-1-}$ mice (Fig. 3). The present study clearly demonstrates that upregulation of PACAP peptide in the dorsal horn, especially in the superficial layer, is crucial for NO formation by nNOS in neuropathic pain mice.

In contrast to the predominantly particulate $\mathrm{Ca}^{2+}$-calmodulin-dependent endothelial NOS, nNOS is mainly soluble, presumably owing to the lack of a posttranslational myristoylation (Hecker et al., 1994). Postsynaptic density protein-95 (PSD-95) was suggested to be required for efficient coupling between NMDA recep-

al., 1993; Narita et al., 1996; Xu and Wiesenfeld-Hallin, 1996; Dickinson and Fleetwood-Walker, 1999). PACAP ${ }^{-1-}$ mice revealed that PACAP, but not VIP, is critical for induction of allodynia by intrathecal administration of NMDA and that the effect of PACAP is biphasic (Fig. $4 B, C$ ). Similarly, biphasic concentration-dependent effects of PACAP on NMDA receptors were found in cortical neurons, with low concentrations $(0.5-2$ $\mathrm{nM}$ ) of PACAP increasing activity, and higher concentrations (10-1000 nM) causing inhibition (Liu and Madsen, 1997). Because $\mathrm{PAC}_{1}$ receptors are constitutively expressed, whether exogenously applied PACAP is anti-nociceptive or pro-nociceptive tor and nNOS (Sattler et al., 1999; Husi and Grant, 2001) and a ternary complex containing nNOS, PSD-95, and NMDA receptor subunit NR2B was identified in the brain (Christopherson et al., 1999). Recently NMDA at $750 \mu \mathrm{M}$ was shown to stimulate NO formation after the translocation of nNOS from the cytosol to the membrane in NGF-differentiated PC12 cells (Arundine et al., 2003). This concentration of $750 \mu \mathrm{M}$ was toxic and induced cell death by continuous exposure. Although membrane association of nNOS to NMDA receptors is considered to occur through PSD-95, an intrinsic factor that stimulates the translocation in the spinal cord was unknown. We demonstrate here that coad- 
ministration of $1 \mathrm{~nm}$ PACAP and $100 \mu \mathrm{M}$ NMDA stimulated the translocation of nNOS from the cytosol to the membrane and NO formation in NGF-differentiated PC12 cells (Fig. 6). Whereas NMDA alone could produce a rapid and transient increase in $\left[\mathrm{Ca}^{2+}\right]_{\mathrm{i}}$ in spinal slices, it could not produce NO (Fig. $5 B)$. Whereas PACAP alone weakly stimulated NO formation, it stimulated NO formation in a dose-dependent manner with 100 $\mu \mathrm{M}$ NMDA (Fig. $5 E$ ). These in vitro experiments are also in line with the increase in nNOS activity revealed by NADPH diaphorase histochemistry observed in the neuropathic mice (Fig. 3G-I) and recovery of NMDA-induced allodynia by intrathecal administration of $1 \mathrm{ng}$ of PACAP in PACAP ${ }^{-1-}$ mice (Fig. $4 \mathrm{~B}$ ).

In addition to a direct modulatory effect of PACAP on the NMDA receptor (Liu et al., 1997), PACAP could potentiate NMDA receptor functions such as expression of brain-derived neurotrophic factor in cortical neurons and astrocytes (Pellegri et al., 1998) and glutamate-evoked release of arachidonic acid from cortical neurons (Stella and Magistretti, 1996). Although a low dose (0.05 nM) of PACAP induced long-lasting facilitation, which was partially blocked by the NMDA receptor antagonist D-AP-5, a high dose $(1 \mu \mathrm{M})$ induced a long-lasting depression of the basal transmission of CA1 synapses in hippocampal slices (Roberto et al., 2001). Furthermore, heterozygous PACAP-deficient mice and $\mathrm{PAC}_{1}{ }^{-1-}$ mice impaired long-term potentiation in the dentate gyrus in vivo (Matsuyama et al., 2003). Whereas long-term potentiation has been extensively studied in the hippocampus and other cortical areas, a similar long-term potentiation and depression in synaptic efficiency can be elicited at primary afferent synapses with neurons in the superficial laminas of the spinal cord (Randić et al., 1993). More than 100 molecules including brain-derived neurotrophic factor and its receptor TrkB, arachidonic acid and its metabolite prostaglandins, and NO have been implicated as mediators or modulators of hippocampal longterm potentiation and spinal central sensitization and subsequent pain hypersensitivity (Ji et al., 2003). Our recent and present studies demonstrate that neuropathic pain after irreversible nerve injury is maintained by reversible NO production in the dorsal horn of the spinal cord. The functional coupling of NMDA receptor and nNOS by upregulation of PACAP is a good example of late-onset, transcriptional-dependent, activitydependent central sensitization (Ji et al., 2003) involved in maintenance of chronic pain. The present study clearly demonstrates that PACAP is a key molecule of pain hypersensitivity in the spinal cord with potential therapeutic relevance for relief of chronic neuropathic pain and possibly nerve repair.

\section{References}

Arundine M, Sanelli T, He BP, Strong MJ (2003) NMDA induces NOS 1 translocation to the cell membrane in NGF-differentiated PC 12 cells. Brain Res 976:149-158.

Chaplan SR, Bach FW, Pogrel JW, Chung JM, Yaksh TL (1994) Quantitative assessment of tactile allodynia in the rat paw. J Neurosci Methods 53:55-63.

Christopherson KS, Hillier BJ, Lim WA, Bredt DS (1999) PSD-95 assembles a ternary complex with the $N$-methyl-D-aspartic acid receptor and a bivalent neuronal NO synthase PDZ domain. J Biol Chem 274:27467-27473.

Dickinson T, Fleetwood-Walker SM (1999) VIP and PACAP: very important in pain? Trends Pharmacol Sci 20:324-329.

Dickinson T, Mitchell R, Robberecht P, Fleetwood-Walker SM (1999) The role of VIP/PACAP receptor subtypes in spinal somatosensory processing in rats with an experimental peripheral mononeuropathy. Neuropharmacology 38:167-180.
Doi Y, Minami T, Nishizawa M, Mabuchi T, Mori H, Ito S (2002) Central nociceptive role of prostacyclin (IP) receptor induced by peripheral inflammation. NeuroReport 13:93-96.

Hashimoto H, Shintani N, Tanaka K, Mori W, Hirose M, Matsuda T, Sakaue M, Miyazaki J, Niwa H, Tashiro F, Yamamoto K, Koga K, Tomimoto S, Kunugi A, Suetake S, Baba A (2001) Altered psychomotor behaviors in mice lacking pituitary adenylate cyclase-activating polypeptide (PACAP). Proc Natl Acad Sci USA 98:13355-13360.

Hashimoto H, Shintani N, Baba A (2002) Higher brain functions of PACAP and homologous Drosophila memory gene amnesiac: insights from knockouts and mutants. Biochem Biophys Res Commun 297:427-431.

Hecker M, Mulsch A, Busse R (1994) Subcellular localization and characterization of neuronal nitric oxide synthase. J Neurochem 62:1524-1529.

Husi H, Grant SGN (2001) Proteomics of the nervous system. Trends Neurosci 24:259-266.

Husi H, Ward MA, Choudhary JS, Blackstock WP, Grant SGN (2000) Proteomic analysis of NMDA receptor-adhesion protein signaling complexes. Nat Neurosci 3:661-669.

Ji RR, Kohno T, Moore KA, Woolf CJ (2003) Central sensitization and LTP: do pain and memory share similar mechanisms? Trends Neurosci 26:696-705.

Jongsma H, Danielsen N, Sundler F, Kanje M (2000) Alteration of PACAP distribution and PACAP receptor binding in the rat sensory nervous system following sciatic nerve transection. Brain Res 853:186-196.

Kind PC, Neumann PE (2001) Plasticity: downstream of glutamate. Trends Neurosci 24:553-555.

Kitanaka J, Hashimoto H, Gotoh M, Kondo K, Sakata K, Hirasawa Y, Sawada M, Suzumura A, Marunouchi T, Matsuda T, Baba A (1996) Expression pattern of messenger RNAs for prostanoid receptors in glial cell cultures. Brain Res 707:282-287.

Kojima H, Urano Y, Kikuchi K, Higuchi T, Hirata Y, Nagano T (1999) Fluorescent indicators for imaging nitric oxide production. Angew Chem Int Ed 38:3209-3212.

Laing I, Todd AJ, Heizmann CW, Schmidt HHHW (1994) Subpopulations of GABAergic neurons in laminae I-III of rat spinal dorsal horn defined by coexistence with classical transmitters, peptides, nitric oxide synthase or parvalbumin. Neuroscience 61:123-132.

Liu GJ, Madsen BW (1997) PACAP $_{38}$ modulates activity of NMDA receptors in cultured chick cortical neurons. J Neurophysiol 78:2231-2234.

Mabuchi T, Matsumura S, Okuda-Ashitaka E, Kitano T, Kojima H, Nagano T, Minami T, Ito S (2003) Attenuation of neuropathic pain by the nociceptin/orphanin FQ antagonist JTC-801 is mediated by inhibition of nitric oxide production. Eur J Neurosci 17:1384-1392.

Matsuyama S, Matsumoto A, Hashimoto H, Shintani N, Baba A (2003) Impaired long-term potentiation in vivo in the dentate gyrus of pituitary adenylate cyclase-activating polypeptide (PACAP) or PACAP type 1 receptor-mutant mice. NeuroReport 14:2095-2098.

Meller ST, Gebhart GF (1993) Nitric oxide (NO) and nociceptive processing in the spinal cord. Pain 52:127-136.

Minami T, Matsumura S, Okuda-Ashitaka E, Shimamoto K, Sakimura K, Mishina M, Mori H, Ito S (2001) Characterization of the glutamatergic system for induction and maintenance of allodynia. Brain Res 895:178-185.

Minami T, Matsumura S, Mabuchi T, Kobayashi T, Sugimoto Y, Ushikubi F, Ichikawa A, Narumiya S, Ito S (2003) Functional evidence for interaction between prostaglandin EP3 and $\kappa$-opioid receptor pathways in tactile pain induced by human immunodeficiency virus type-1 (HIV-1) glycoprotein gp120. Neuropharmacology 45:96-105.

Moller K, Zhang YZ, Håkanson R, Luts A, Sjölund B, Uddman R, Sundler F (1993) Pituitary adenylate cyclase activating peptide is a sensory neuropeptide: immunocytochemical and immunochemical evidence. Neuroscience 57:725-732.

Narita M, Dun SL, Dun NJ, Tseng LF (1996) Hyperalgesia induced by pituitary adenylate cyclase-activating polypeptide in the mouse spinal cord. Eur J Pharmacol 311:121-126.

Pellegri G, Magistretti PJ, Martin JL (1998) VIP and PACAP potentiate the action of glutamate on BDNF expression in mouse cortical neurones. Eur J Neurosci 10:272-280.

Randić M, Jiang MC, Cerne R (1993) Long-term potentiation and long- 
term depression of primary afferent neurotransmission in the rat spinal cord. J Neurosci 13:5228-5241.

Roberto M, Scuri R, Brunelli M (2001) Differential effects of PACAP-38 on synaptic responses in rat hippocampal CA1 region. Learn Mem 8:265-271.

Sattler R, Xiong Z, Lu WY, Hafner M, MacDonald JF, Tymianski M (1999) Specific coupling of NMDA receptor activation to nitric oxide neurotoxicity by PSD-95 protein. Science $284: 1845-1848$.

Sheehy AM, Phung YT, Riemer RK, Black SM (1997) Growth factor induction of nitric oxide synthase in rat pheochromocytoma cells. Mol Brain Res 52:71-77.

Stella N, Magistretti PJ (1996) Vasoactive intestinal peptide (VIP) and pituitary adenylate cyclase-activating polypeptide (PACAP) potentiate the glutamate-evoked release of arachidonic acid from mouse cortical neurons. J Biol Chem 271:23705-23710.

Vaudry D, Vaudry D, Gonzalez BJ, Basille M, Yon L, Fournier A, Vaudry H (2000) Pituitary adenylate cyclase-activating polypeptide and its receptors: from structure to functions. Pharmacol Rev 52:269-324.

Xu XJ, Wiesenfeld-Hallin Z (1996) Intrathecal pituitary adenylate cyclase activating polypeptide facilitates the spinal nociceptive flexor reflex in the rat. Neuroscience 72:801-804.

Zhang Q, Shi TJ, Ji RR, Zhang YZ, Sundler F, Hannibal J, Fahrenkrug J, Hökfelt T (1995) Expression of pituitary adenylate cyclase-activating polypeptide in dorsal root ganglia following axotomy: time course and coexistence. Brain Res 705:149-158.

Zhang Y, Danielsen N, Sundler F, Mulder H (1998) Pituitary adenylate cyclase-activating peptide is upregulated in sensory neurons by inflammation. NeuroReport 9:2833-2836.

Zhang YZ, Sjölund B, Moller K, Håkanson R, Sundler F (1993) Pituitary adenylate cyclase activating peptide produces a marked and long-lasting depression of a C-fibre-evoked flexion reflex. Neuroscience 57:733-737.

Zhang YZ, Hannibal J, Zhao Q, Moller K, Danielsen N, Fahrenkrug J, Sundler F (1996) Pituitary adenylate cyclase activating peptide expression in the rat dorsal root ganglia: up-regulation after peripheral nerve injury. Neuroscience 74:1099-1110.

Zimmermann M (1983) Ethical guidelines for investigations of experimental pain in conscious animals. Pain 16:109-110. 